\title{
Optimal Multigrid Algorithms for the Massive Gaussian Model and Path Integrals
}

\author{
A. Brandt ${ }^{1}$ and M. Galun ${ }^{1}$
}

Received June 19, 1995; final July 25, 1995

\begin{abstract}
Multigrid algorithms are presented which, in addition to eliminating the critical slowing down, can also eliminate the "volume factor". The elimination of the volume factor removes the need to produce many independent fine-grid configurations for averaging out their statistical deviations, by averaging over the many samples produced on coarse grids during the multigrid cycle. Thermodynamic limits of observables can be calculated to relative accuracy $\varepsilon_{r}$ in just $O\left(\varepsilon_{r}^{-2}\right)$ computer operations, where $\varepsilon_{r}$ is the error relative to the standard deviation of the observable. In this paper, we describe in detail the calculation of the susceptibility in the one-dimensional massive Gaussian model, which is also a simple example of path integrals. Numerical experiments show that the susceptibility can be calculated to relative accuracy $\varepsilon_{r}$ in about $8 \varepsilon_{r}^{-2}$ random number generations, independent of the mass size.
\end{abstract}

KEY WORDS: Multigrid; massive Gaussian model; Monte Carlo; critical slowing down; volume factor; thermodynamic limit; path integrals.

\section{INTRODUCTION}

One of the aims in statistical physics is to calculate various average properties of configurations governed by the Boltzmann distribution. This is usually done by measuring these averages over a sequence of Monte Carlo iterations. Unfortunately, such processes tend to suffer from several independent inefficiency factors that multiply each other and thus produce very expensive computations.

The best known of these inefficiency factors is the critical slowing down (CSD). This is the phenomenon, typical of simulations of critical systems,

\footnotetext{
${ }^{1}$ Department of Applied Mathematics and Computer Science, Weizmann Institute of Science, Rehovot 76100, Israel.
} 
that with the increase in lattice size there also comes an increase in the number of full Monte Carlo passes over the lattice needed to produce a new configuration which is statistically "useful", i.e., substantially independent of, or only weakly correlated to, a former configuration. Considerable effort has been devoted to reducing the critical slowing down. For simple cases with real variables, classical multigrid methods can eliminate the CSD. For more complicated models (e.g., $\phi^{4}$; or discrete models) more recent publications report on simulation techniques that partially or completely $^{(1,8,9,13)}$ eliminate the CSD. This means that the time to produce an independent configuration is proportional to the number of gridpoints.

The elimination of the CSD is very important, but there is another no less important factor of slowness, the volume factor. To calculate a thermodynamic quantity to a certain relative accuracy $\varepsilon_{r}$, one needs to produce $O\left(\varepsilon_{r}^{-2}\right)$ essentially independent configurations to average out the deviation exhibited by each of them, where the relative accuracy $\varepsilon_{r}$ is the error relative to the standard deviation of the observable in question. Also, the size of the grid must increase as some positive power of $\varepsilon_{r}^{-1}$. Thus, even if the CSD has been completely eliminated, the overall work increases as $O\left(\varepsilon_{r}^{-2} N^{d}\right)$, where $N$ is the linear lattice size and $d$ is the dimension. An important advantage of the multigrid approach is that it can drastically reduce the volume factor $N^{d}$ as well, by averaging over many samples produced in prolonged Monte Carlo passes on coarse grids. Indeed, we will exhibit cases in which the volume factor is completely eliminated together with the CSD.

The elimination of both the volume factor and the CSD means that a thermodynamic limit can be calculated to an accuracy of $\pm \varepsilon$ in optimal time, i.e., in only $O\left(\varepsilon^{-2}\right)$ computer operations. This is just the same order of complexity as needed to calculate, by statistical trials, any simple "pointwise" average, such as the frequency of "heads" in coin tossing. By contrast, both the volume and the CSD factors multiply the statistical factor $\left(\varepsilon^{-2}\right)$ in the operation count of conventional algorithms.

The elimination of the volume factor was first been demonstrated ${ }^{(3,4,6)}$ for the Gaussian model with constant coefficients. It was shown there, for the one-dimensional Gaussian model, that the susceptibility can be calculated to accuracy $\varepsilon_{r}$ in about $4 \varepsilon_{r}^{-2}$ random number generations, while the average energy per degree of freedom requires $3 \varepsilon_{r}^{-2}$ such generations for a similar accuracy. In the two-dimensional Gaussian model, the susceptibility can be measured to accuracy $\varepsilon_{r}$ in about $20 \varepsilon_{r}^{-2}$ random number generations.

In this paper we treat the one-dimensional massive Gaussian model and we show that, using an appropriate multigrid algorithm, one can calculate the susceptibility in an optimal time. Stated differently, we show 
where the Fourier coefficient $c_{j}$ are real. The magnetization is given by

$$
M(u)=\frac{1}{L} \int_{0}^{L} u(x) d x=\frac{2}{\pi} \sum_{j=1}^{\infty} * \frac{c_{j}}{j}
$$

where $\Sigma^{*}$, here and below, stands for a summation over odd integers. The probability density of each configuration $u$ is given by the density function of the Boltzmann distribution

$$
P(u)=\frac{e^{-\mathscr{H}(u) / T}}{Z(T)}
$$

where $T$ is the temperature and $Z(T)$ is a normalization factor. It can be shown (see Appendix A) that the average magnetization $\langle M\rangle$ and the susceptibility $\left\langle M^{2}\right\rangle-\langle M\rangle^{2}$ are given by

$$
\begin{aligned}
\langle M\rangle & =0 \\
\left\langle M^{2}\right\rangle-\langle M\rangle^{2} & =\left\langle M^{2}\right\rangle=\frac{4 L T}{\pi^{2}} \sum_{j=1}^{\infty} \frac{1}{\pi^{2} j^{4}+m^{2} L^{2} j^{2}}
\end{aligned}
$$

We define any statistics for the continuum as the limit of the statistics for systems truncated to a finite number of Fourier components.

\subsection{Discrete Case}

In order to measure such statistical averages numerically, it is necessary to discretize the system. On a grid with meshsize $h=L / N$, the discretized Hamiltonian $\mathscr{H}_{h}(u)$ approximating (1) can be written as

$$
\mathscr{H}_{h}(u)=\frac{1}{h} \sum_{i=1}^{N}\left(u_{i}-u_{i-1}\right)^{2}+m^{2} h \sum_{i=1}^{N-1} u_{i}^{2}
$$

where $u_{i}=u\left(x_{i}\right)$ are the variables at gridpoints $x_{i}=i h, 0 \leqslant i \leqslant N$, respectively. For the simplicity of the multigrid algorithm we assume $N=2^{k}$. Assuming again zero boundary conditions, $u_{0}=u_{N}=0$, we can represent a general grid configuration by

$$
u_{i}=\sum_{j=1}^{N-1} c_{j} \sin \left(j \pi x_{i} / L\right)
$$

The discrete magnetization is given by

$$
M_{h}(u)=\frac{h}{L} \sum_{i=0}^{N} u\left(x_{i}\right)=\frac{h}{L} \sum_{j=1}^{N-1} c_{j} \frac{\cos [j \pi h /(2 L)]}{\sin [j \pi h /(2 L)]}
$$



where $\mathscr{H}_{h}\left(\tilde{u}^{h}\right)$ is given by $(10)$ and $\mathscr{H}_{H}\left(u^{H}\right)$ is

$$
\begin{aligned}
\mathscr{H}_{H}\left(u^{H}\right)= & \frac{1}{H} \sum_{I=1}^{N / 2}\left(u_{I}^{H}-u_{I-1}^{H}\right)^{2}+H \sum_{I=1}^{N / 2-1} \phi_{I}^{H} u_{I}^{H} \\
& +m^{2} H b_{H} \sum_{I=1}^{N-1}\left(u_{I}^{H}\right)^{2}+m^{2} H a_{H} \sum_{I=1}^{N-1} u_{I}^{H} u_{I-1}^{H}
\end{aligned}
$$

with

$$
a_{H}=\frac{a_{h}}{2}+\frac{b_{h}}{4}, \quad b_{H}=\frac{a_{h}}{2}+\frac{3 b_{h}}{4}
$$

and

$$
\begin{aligned}
\phi_{I}^{H}= & \frac{-\tilde{u}_{i-2}^{h}+2 \tilde{u}_{i}^{h}-\tilde{u}_{i+2}^{h}}{2 h^{2}}+\frac{\phi_{i-1}^{h}+2 \phi_{i}^{h}+\phi_{i+1}^{h}}{4} \\
+ & \frac{m^{2} b_{h}}{2}\left(\tilde{u}_{i-1}^{h}+2 \tilde{u}_{i}^{h}+\tilde{u}_{i+1}^{h}\right)+\frac{m^{2} a_{h}}{4}\left(\tilde{u}_{i-2}^{h}+2 \tilde{u}_{i-1}^{h}+2 \tilde{u}_{i}^{h}+2 \tilde{u}_{i+1}^{h}+\tilde{u}_{i+2}^{h}\right) \\
& (I=i / 2=1, \ldots, N / 2-1)
\end{aligned}
$$

representing fine-to-coarse induced field-like terms. The coefficients $a^{H}$ and $b^{H}$ depend only on $a^{h}$ and $b^{h}$. The coarse field terms $\phi_{I}^{H}$ are calculated from the details of the fine-grid configuration at coarsening and are fixed throughout the processing on the coarser level. The variables of the coarse grid $u_{I}^{H}$ are initially set to zero, corresponding to zero initial displacements.

Having calculated the field $\phi^{H}$ once for all, $\mathscr{H}_{H}$ is directly calculated in terms of the coarse-grid configuration $u^{H}$, there is no need to explicitly perform (12) in order to relax the coarser level. One can therefore run a long Monte Carlo process with $\mathscr{H}_{H}$, (13), before explicitly updating $u^{h}$ by (11).

The entire algorithm can be described by a sequence of multigrid cycles for the finest level. A cycle for any given ("current") level is recursively defined by the following five steps:

1. First make $v_{1}$ Monte Carlo sweeps on the current level. Then, if this level is the coarsest, go to 5 .

2. Create the next coarser level from the current one by determining the coefficients (14a) and the coarse field-like terms (14b). 
3. Perform $\gamma$ multigrid cycles for the coarse level. ( $\gamma$ may change from the current level to another.)

4. Update the current level by performing (11).

5. Finally, make additional $v_{2}$ Monte Carlo sweeps on the current level.

The Monte Carlo sweeps are performed by changing each variable in its turn randomly according to its associated distribution, regarding its neighbors as fixed.

The values of $v_{1}, v_{2}$ and $\gamma$ are discussed below?

The massive Gaussian model displays criticality as $m \rightarrow 0$. The described cycle, even with $\gamma=1$ (a $V$-cycle), would eliminate the critical slowing down, but the volume factor remains intact. However, the main issue here is to eliminate the volume factor as well, for any mass size $m$; the way to do so is described next.

\subsection{Fast Sampling of Susceptibility}

As in the simple Gaussian model, the susceptibility $(5 b)$ is dominated by contributions from large-scale fluctuations (low-frequency Fourier components), regardless of the size $m^{2}$. Therefore, the purpose of the simulation is to sample quickly as many such fluctuations as possible. The way to do this is to use a cycle index $\gamma$ larger than 1 and to calculate the susceptibility over the many measurements on the coarsest level. Furthermore, the optimal multigrid algorithm differs from the one that has been described for the simple Gaussian model $;^{(3,4,6)}$ the cycle index may change from one level to another, depending on the parameter $m$.

The magnetization $M_{h}$ can be evaluated on any level [plug (11) in (8) ], without going back to finer levels. Thus, many measurements of $M_{h}^{2}$ can be made within a cycle, and their average $\overline{M_{h}^{2}}$ can be used as an estimate for the discrete susceptibility $\left\langle M_{h}^{2}\right\rangle$. In practice, measurements are taken only on the coarsest level, after each relaxation sweep there, since only there are substantial changes in $M_{h}$ introduced.

We next study the number $s_{i}$ of relaxation sweeps the algorithm needs to perform on level $i$, i.e., on the grid with mesh size $h_{i}=2^{i} h[i=0,1, \ldots$, $\left.\ell=\log _{2}(N / 2)\right]$, in order to achieve accuracy $\varepsilon$ in the estimation of the susceptibility. The total expected error $\varepsilon$ in measuring $\left\langle M^{2}\right\rangle$ is calculated by Fourier analysis in Appendix B. From (B2), the total error in measuring $\left\langle M^{2}\right\rangle$ relative to the standard deviation $\sigma$, where

$$
\sigma=\left(\left\langle M^{4}\right\rangle-\left\langle M^{2}\right\rangle^{2}\right)^{1 / 2}=\sqrt{2}\left\langle M^{2}\right\rangle=O\left(\frac{L T}{\pi^{2}+m^{2} L^{2}}\right)
$$


is

$$
\varepsilon_{r}=\frac{\varepsilon}{\sigma}=O\left(\sum_{i=0}^{l} s_{i}^{-1 / 2} h_{i}^{3} L^{-3} \frac{\pi^{2}+m^{2} L^{2}}{\pi^{2}+m^{2} h_{i}^{2}}\right)+\text { r.d.e. }
$$

where the last term added here (r.d.e) is the relative discretization error estimator which is discussed in Appendix C. It is important to emphasize here that raising $p$, the order of the discretization error, beyond $p=3$ has no benefit. [See (C1). This point, incidentally, was missed in ref. 4.]

The total work (operations) on all the levels is clearly

$$
W=\sum_{i=0}^{l} s_{i} O\left(L / h_{i}\right)
$$

The optimal choice for $s_{i}$ (yielding either minimal $\varepsilon$ for a given $W$ or minimal $W$ for a given $\varepsilon$ ) is obtained when $\partial \varepsilon_{r} / \partial s_{i}+\lambda_{1} \partial W / \partial s_{i}=0$, which by (15) and (16) yields

$$
s_{i}=\lambda_{2}\left(L^{-4} h_{i}^{4} \frac{\pi^{2}+m^{2} L^{2}}{\pi^{2}+m^{2} h_{i}^{2}}\right)^{2 / 3}=\lambda_{3} 2^{8 i / 3}\left(\frac{\pi^{2}+m^{2} L^{2}}{\pi^{2}+m^{2} h_{i}^{2}}\right)^{2 / 3}
$$

where $\lambda_{1}, \lambda_{2}$ and $\lambda_{3}$ are independent of $i$. Hence, the optimal cycle index at level $i$ is

$$
\gamma_{i_{\mathrm{opt}}}=\frac{s_{i+1}}{s_{i}}=2^{8 / 3}\left(\frac{\pi^{2}+m^{2} h_{i}^{2}}{\pi^{2}+4 m^{2} h_{i}^{2}}\right)^{2 / 3}
$$

The actual values of (17) for constructing an optimal multigrid cycle are given in Table I as a function of $m h_{i}$. However, we will see in the experiments that the results are not much sensitive to changes of $\gamma$ within quite large margins. In fact, analyzing the following three cases would show wide ranges of $\gamma$ at which the optimal order $W=O\left(\varepsilon_{r}^{-2}\right)$ is still obtained. For each of the cases we will use fixed $\gamma$, hence $s_{i}=s \gamma^{i}$, where $s=$ $\left(v_{1}+v_{2}\right) \cdot$ \# cycles. Since $h_{i} L^{-1}=O\left(2^{i-l}\right)$, we can perform the summations in (15) and (16). Using the discussion in Appendix $\mathrm{C}$ and the relation $\sigma=O\left(L T /\left(\pi^{2}+m^{2} L^{2}\right)\right)$, we can calculate the general relative discretization error in each of the three cases:

1. For the case $h_{i}<\pi / m$ at all levels $(i=0, \ldots, l)$

$$
\varepsilon_{r}=O\left(s^{-1 / 2} \frac{\gamma^{-l / 2}-2^{-3 l}}{1-2^{-3} \gamma^{1 / 2}}\right)+O\left(2^{-l p_{*}}\right)
$$

and

$$
W=O\left(s \frac{\gamma^{l}-2^{l}}{1-2 \gamma^{-1}}\right)
$$


Table I. Constructing an Optimal Multigrid Cycle ${ }^{a}$

\begin{tabular}{lcc}
\hline & & Practical \\
$m h_{i}$ & $\gamma_{i_{\mathrm{opt}}}$ & $\gamma_{i_{\mathrm{opt}}}$ \\
\hline$\ll 1$ & 6.35 & 6 \\
0.5 & 6.05 & 6 \\
1 & 5.40 & 5 \\
2 & 4.19 & 4 \\
4 & 3.15 & 3 \\
8 & 2.70 & 3 \\
$\gg 1$ & 2.52 & 3 \\
\hline
\end{tabular}

\footnotetext{
${ }^{a}$ The table gives the optimal cycle index $\gamma_{i_{\mathrm{opt}}}$ at level $i$ as a function of $m h_{i}$ (the mass size times the meshsize at level $i$ ).
}

for any $2<\gamma<2^{6}$, where $p_{*}=\min (3, p)$. Actually, by choosing $\gamma$ and the approximation order $p$ so that $\gamma$ is significantly smaller than $2^{2 p_{*}}$, we can ignore the second term in (18), yielding $W=O\left(\varepsilon_{r}^{-2}\right)$. While $\gamma=\gamma_{\text {opt }}$ indeed minimizes $W \varepsilon_{r}^{2}$, the other cycle indices $\left(2<\gamma<2^{2 p_{*}}\right)$ give practically the same efficiency. This case is very similar to the simple Gaussian case. ${ }^{(4)}$

2. For the case $h_{i}>\pi / m$ at all levels $(i=0, \ldots, l)$

$$
\varepsilon_{r}=O\left(s^{-1 / 2} \frac{\gamma^{-l / 2}-2^{-l}}{1-2^{-1} \gamma^{1 / 2}}\right)+O\left(2^{-l}\right)
$$

and $W$ is as before (19), for any $2<\gamma<4$. As any $\gamma$ in this range is already smaller than $2^{2}$, the second term in (20) can be ignored, yielding again $W=O\left(\varepsilon_{r}^{-2}\right)$.

3. As $h \rightarrow 0$ the last case will evolve eventually to the case $h_{i}<\pi / m$ for $i=0,1, \ldots, k-1$ and $h_{i}>\pi / m$ for $i=k, k+1, \ldots, l$. Generally, in this case

$$
\begin{aligned}
\varepsilon_{r}= & O\left(s^{-1 / 2} \gamma^{-l / 2} \sum_{j=0}^{\hat{k}}\left(2^{-1} \gamma^{1 / 2}\right)^{j}\right) \\
& +O\left(s^{-1 / 2} \gamma^{-l / 2} \frac{1-\left(2^{-3} \gamma^{1 / 2}\right)^{k}}{1-2^{-3} \gamma^{1 / 2}} m^{2} L^{2}\left(2^{-3} \gamma^{1 / 2}\right)^{\hat{k}}\right) \\
& +O\left(2^{-l p_{*}}\right)
\end{aligned}
$$

and $W$ is as before (19), for any $2<\gamma<2^{6}$, where $\hat{k}=l-k$ stays constant as $h \rightarrow 0$. As mentioned earlier, a multigrid cycle as described in Table I 
indeed minimizes $W \varepsilon_{r}^{2}$, but by choosing any fixed $\gamma$ in the domain $\left(2<\gamma<2^{2 p_{*}}\right)$, we can neglect the third term in (21), again yielding the optimal efficiency $W=O\left(\varepsilon_{r}^{-2}\right)$.

\subsection{Numerical Results}

We have tested the multigrid algorithm for different values of $m$ with grid of sizes up to 512. Our main aim was to show that using appropriate values of $\gamma$ one can calculate the susceptibility in an optimal time, while the use of unsuitable values of $\gamma$ undermines optimality. The susceptibility has been measured over just one cycle. Within the cycle, many measurements are taken, in fact after each Monte Carlo step on the coarsest level, the level with just one internal point, i.e., $h_{l}=L / 2$. The average of the measurements $\overline{M_{h}^{2}}$ is an approximation for $\left\langle M_{h}^{2}\right\rangle,(9 \mathrm{~b})$, which is also an approximation for the thermodynamic limit $\left\langle M^{2}\right\rangle$, (5b). The relative accuracy is defined as $\varepsilon_{r}=\left|\overline{M_{h}^{2}}-\left\langle M^{2}\right\rangle\right| / \sigma$ and it is averaged over an ensemble of 10,000 runs. $^{2}$ We define $\alpha$ to be the expected value of \#RAN $\cdot \varepsilon_{r}^{2}$, where \#RAN is the amount of work spent in the cycle, measured by the number of times a random number is generated. Thus, $\alpha$ should turn out to be a constant if and only if the algorithm solves to relative accuracy $\varepsilon_{r}$ in $O\left(\varepsilon_{r}^{-2}\right)$ operations. We measured $\alpha$ for three different cases. Results are presented in Table II for $L=1, T=1, h_{0}=1 / N$ and $h_{l}=1 / 2$, showing that the algorithm is not sensitive in a wide range of suitable $\gamma$. We see that any appropriate cycle index will lead to the optimal efficiency, i.e., $\alpha$ tends to a constant as $N$ grows (see cases $m=0.5$, $\sigma=0.05749$ and $m=64, \sigma=1.672 \times 10^{-4}$ ). In the last case, $m=400, \sigma=$ $4.397 \times 10^{-6}, \alpha$ turns out to be a constant when cycle index 3 is used, but cycle index 6 , as explained above, is too big for this case. For any case, cycle index 2 ( $W$-cycle) is below the optimal range, demonstrating logarithmic growth of $\alpha$. The main conclusion is that an optimal algorithm, with practically constant $\alpha$, can always be devised.

In Table III, we compare our optimal multigrid Monte Carlo algorithm and a conventional multigrid algorithm, where the susceptibility is measured once per $V$-cycle. ${ }^{(5)}$ It is clear that better accuracy means using larger grids. Therefore, as the accuracy is improved, the ratio between the complexity of the two algorithms increases. For example, in order to achieve a certain accuracy in the case $m=64$ and $N=512$, it would cost a conventional algorithm 330 times the work required by the optimal multigrid algorithm as presented here. Practically, while the computational time

${ }^{2}$ The experiments for $m=64$ and $N=512$ using Table I and $\gamma=6$ are made over an ensemble of 4000 runs and 400 runs, respectively. 
Table II. Performance in Measuring Susceptibility ${ }^{a}$

\begin{tabular}{cccccccccc}
\hline & & \multicolumn{10}{c}{$\alpha$} \\
\cline { 3 - 9 }$m$ & $\gamma$ & $N=4$ & $N=8$ & $N=16$ & $N=32$ & $N=64$ & $N=128$ & $N=256$ & $N=512$ \\
\hline \multirow{2}{*}{0.5} & 2 & 2.4 & 5 & 7.3 & 9.8 & 12.3 & 14.6 & 17.4 & \\
& 3 & 2.2 & 3.5 & 4.5 & 5.1 & 5.3 & 5.9 & & \\
& 6 & 2 & 3 & 3.6 & 3.8 & 3.9 & 4 & & \\
& 7 & 2 & 2.8 & 3.5 & 3.7 & 3.8 & 3.8 & & \\
64 & Table I & 1.6 & 2.9 & 4.2 & 5.3 & 5.9 & 5.9 & 6.3 & 6.2 \\
& 3 & 1.6 & 2.9 & 4.2 & 5.3 & 6.1 & 6.5 & 6.7 & 6.8 \\
& 6 & 1.5 & 2.6 & 4.2 & 5.8 & 6.8 & 7.7 & 7.8 & 8.4 \\
400 & 2 & 1.8 & 3.9 & 6.4 & 9.7 & 12.8 & 15.5 & 18.6 & \\
& 3 or Table I & 1.6 & 2.9 & 4.3 & 5.6 & 6.8 & 7.5 & 8 & \\
& 6 & 1.5 & 2.8 & 4.9 & 7.7 & 11.2 & 16.2 & 20 & \\
\hline
\end{tabular}

${ }^{a}$ The table shows $\alpha$, the number of random generations times the square of the obtained relative accuracy, for the indicated values of the system size $N$ and the cycle index $\gamma$.

of the conventional algorithm ${ }^{(5)}$ is $4 N \varepsilon_{r}^{-2}$ (the cost of conventional algorithms as described in refs. 7 and 10 would be even somewhat bigger), the computational time of our algorithm is about $8 \varepsilon_{r}^{-2}$, independent of the gridsize $N$. [Note that, for maximal efficiency, a conventional algorithm should use the smallest possible $N$ which still gives r.d.e. comparable to $\varepsilon_{r}$. According to Appendix $\mathrm{C}$, this would mean $N=O\left(\varepsilon^{-1 / p^{*}}\right)$.]

Table III. Computational Time (in units of $\varepsilon_{r}^{-2}$ ) in Measuring the Susceptibility on a Grid with $N$ Gridpoints to Relative Accuracy $\varepsilon_{r}{ }^{a}$

\begin{tabular}{|c|c|c|c|c|c|c|c|c|c|}
\hline \multirow[b]{2}{*}{$m$} & \multirow[b]{2}{*}{$\begin{array}{l}\text { Multigrid } \\
\text { algorithm }\end{array}$} & \multicolumn{8}{|c|}{ Computational time } \\
\hline & & $N=4$ & $N=8$ & $N=16$ & $N=32$ & $N=64$ & $N=128$ & $N=256$ & $N=512$ \\
\hline \multirow[t]{2}{*}{0.5} & Conventional & 6.8 & 21.8 & 50.5 & 112.2 & 237 & 484.6 & & \\
\hline & Optimal & 2 & 2.8 & 3.5 & 3.7 & 3.8 & 3.8 & & \\
\hline \multirow[t]{2}{*}{64} & Conventional & 4.9 & 17.4 & 52.6 & 115.6 & 236.7 & 485.2 & 992.9 & 2048.3 \\
\hline & Optimal & 1.6 & 2.9 & 4.2 & 5.3 & 5.9 & 5.9 & 6.3 & 6.2 \\
\hline \multirow[t]{2}{*}{400} & Conventional & 4.7 & 16.8 & 50.6 & 112.2 & 235.1 & 501.9 & 960.9 & \\
\hline & Optimal & 1.6 & 2.9 & 4.3 & 5.6 & 6.8 & 7.5 & 8 & \\
\hline
\end{tabular}

${ }^{a}$ Conventional multigrid method (one measurement per cycle, as in refs. 5, 7, and 10) vs. our optimal multigrid method. 


\section{SUMMARY}

The calculation of a thermodynamic limit of any observable to a relative accuracy $\varepsilon_{r}$ usually requires by a Monte Carlo process

$$
O\left(N^{d+z} \varepsilon_{r}^{-2}\right)
$$

computer operations, where $\varepsilon_{r}$ is the error relative to the standard deviation of the observable, $N$ is the linear dimension of the lattice needed to approximate the thermodynamic limit to accuracy $\varepsilon_{r}, d$ is the dimension, and $z$ is the critical exponent.

Multigrid algorithms potentially can reduce and even eliminate not only the critical slowing down factor $N^{z}$ but also the volume factor $N^{d}$.

The parameters of the multigrid algorithm, such as the cycle index $\gamma$ and the coarse-to-fine interpolation order, depends not only on the involved model and its discretization, but also the observable in question. For the optimal calculation of the susceptibility in the one-dimensional massive Gaussian model it is essential to use linear interpolation and a cycle index which varies with the mass size. In this case the critical slowing down and the volume factor are completely eliminated leading to the optimal efficiency $O\left(\varepsilon_{r}^{-2}\right)$.

\section{APPENDIX A. FOURIER TRANSFORM EXPRESSIONS}

In the continuous case, by substituting (2) into (1) and into the left part of (3), one gets

$$
\mathscr{H}(u)=\frac{\pi^{2}}{2 L} \sum_{j=1}^{\infty} j^{2} c_{j}^{2}+\frac{m^{2} L}{2} \sum_{j=1}^{\infty} c_{j}^{2}
$$

and the right hand side of (3). From (4) and (A1), it can be shown by straightforward calculations that

$$
\begin{aligned}
& \left\langle c_{j}\right\rangle=0 \\
& \left\langle c_{j}^{2}\right\rangle=\frac{L T}{\pi^{2} j^{2}+m^{2} L^{2}} \\
& \left\langle c_{j}^{4}\right\rangle=\frac{3 L^{2} T^{2}}{\left(\pi^{2} j^{2}+m^{2} L^{2}\right)^{2}}
\end{aligned}
$$

Hence, the average magnetization $\langle M\rangle$ and the susceptibility $\left\langle M^{2}\right\rangle-\langle M\rangle^{2}$ can be calculated using (3), (A2), and (A3), leading to results (5a) and (5b) in Section 2.1. 
In the discrete case, by substituting (7) into (6) and into the left part of (8) one gets

$$
\mathscr{H}_{h}(u)=\frac{2 L}{h^{2}} \sum_{j=1}^{N-1} c_{j}^{2} \sin ^{2}\left(\frac{j \pi h}{2 L}\right)+\frac{m^{2} L^{N}}{2} \sum_{j=1}^{N-1} c_{j}^{2}
$$

and the right-hand side of (8). From (4) and (A5) we can derive

$$
\begin{aligned}
\left\langle c_{j}\right\rangle & =0 \\
\left\langle c_{j}^{2}\right\rangle & =\frac{T h^{2}}{4 L \sin ^{2}[j \pi h /(2 L)]+m^{2} L h^{2}}
\end{aligned}
$$

The average discrete magnetization (9a) and the discrete susceptibility (9b) in Section 2.2 are obtained by applying (A6) and (A7) to (8).

\section{APPENDIX B. FOURIER ANALYSIS OF THE EXPECTED ERROR IN THE ESTIMATION OF THE SUSCEPTIBILITY}

The relaxation sweep on level $i$ [with meshsize $h_{i}=2^{i} h: i=0,1, \ldots, \ell=$ $\left.\log _{2}(N / 2)\right]$ strongly affects, hence effectively samples, only those Fourier coefficients $c_{j}$ [cf. (2)] for which $j=O\left(L / h_{i}\right)$. Hence, the number $s_{i}$ of relaxation sweeps needed to be performed on level $i$ depends on the contribution of these components to the deviations in measuring $\left\langle M^{2}\right\rangle$. By (3)

$$
M^{2}=\frac{4}{\pi^{2}} \sum_{j, k}^{*} \frac{c_{j} c_{k}}{j k}
$$

Consider first a term $(j, k)$ in (B1) for which both $j$ and $k$ are $O\left(L / h_{i}\right)$, hence the term is effectively sampled $O\left(s_{i}\right)$ times in a cycle. According to (A2)-(A4) in Appendix A, the standard deviation of the term is

$$
\frac{4}{j k \pi^{2}}\left(\left\langle\left(c_{j} c_{k}\right)^{2}\right\rangle-\left\langle c_{j} c_{k}\right\rangle^{2}\right)^{1 / 2}=O\left(h_{i}^{4}\left(\pi^{2}+m^{2} h_{i}^{2}\right)^{-1} L^{-3} T\right)
$$

hence the standard deviation of its average over the $O\left(s_{i}\right)$ samples is $O\left(s_{i}^{-1 / 2} h_{i}^{4}\left(\pi^{2}+m^{2} h_{i}^{2}\right)^{-1} L^{-3} T\right)$. There are $O\left(h_{i}^{-2} L^{-2}\right)$ such terms, where each pair of them is uncorrelated, hence their total contribution is

$$
O\left(s_{i}^{-1 / 2} h_{i}^{3}\left(\pi^{2}+m^{2} h_{i}^{2}\right)^{-1} L^{-2} T\right)
$$

In the case $j=O\left(L / h_{i-r}\right)$ and $k=O\left(L / h_{i}\right)$, where $r \geqslant 1$ (i.e., $h_{i}>h_{i-r}$ ), the term $(j, k)$ in (B1) is effectively sampled as follows (see also ref. 4 ): in 
an inner loop, for a (nearly) fixed value of $c_{j}$, the values of $c_{k}$ are averaged $O\left(s_{i} / s_{i-r}\right)$ times, yielding an average whose deviation is of the order

$$
O\left(\frac{c_{j}}{j k}\left(\frac{s_{i}}{s_{i-r}}\right)^{-1 / 2}\left\langle c_{k}^{2}\right\rangle^{1 / 2}\right)=O\left(\frac{c_{j}}{j k}\left(\frac{s_{i-r}}{s_{i}} \cdot \frac{L T}{\pi^{2} k^{2}+m^{2} L^{2}}\right)^{1 / 2}\right)
$$

Then, in an outer loop, the $c_{j}$ in this average is averaged over $O\left(s_{i-r}\right)$ samples, giving results with deviations of order

$$
\begin{aligned}
& O\left(\frac{s_{i-r}^{-1 / 2}}{j k}\left(\frac{s_{i-r}}{s_{i}} L T\right)^{1 / 2}\left(\pi^{2} k^{2}+m^{2} L^{2}\right)^{-1 / 2}\left\langle c_{j}^{2}\right\rangle^{1 / 2}\right) \\
& =O\left(\frac{s_{i}^{-1 / 2}}{j k}\left(\pi^{2} k^{2}+m^{2} L^{2}\right)^{-1 / 2}\left(\pi^{2} j^{2}+m^{2} L^{2}\right)^{-1 / 2} L T\right) \\
& =O\left(s_{i}^{-1 / 2} h_{i}^{2} h_{i-r}^{2}\left(\pi^{2}+m^{2} h_{i}^{2}\right)^{-1 / 2}\left(\pi^{2}+m^{2} h_{i-r}^{2}\right)^{-1 / 2} L^{-3} T\right)
\end{aligned}
$$

There are $O\left(h_{i}^{-1} h_{i-r}^{-1} L^{2}\right)$ such terms, effectively uncorrelated, hence their total deviation is

$$
O\left(s_{i}^{-1 / 2} h_{i}^{3 / 2} h_{i-r}^{3 / 2}\left(\pi^{2}+m^{2} h_{i}^{2}\right)^{-1 / 2}\left(\pi^{2}+m^{2} h_{i-r}^{2}\right)^{-1 / 2} L^{-2} T\right)
$$

Summing over integers $r \geqslant 0$ gives again

$$
\begin{gathered}
O\left(s_{i}^{-1 / 2} h_{i}^{3 / 2}\left(\pi^{2}+m^{2} h_{i}^{2}\right)^{-1 / 2} T L^{-2} \sum_{r \geqslant 0} h_{i-r}^{3 / 2}\left(\pi^{2}+m^{2} h_{i-r}^{2}\right)^{-1 / 2}\right) \\
=O\left(s_{i}^{-1 / 2} h_{i}^{3}\left(\pi^{2}+m^{2} h_{i}^{2}\right)^{-1} L^{-2} T\right)
\end{gathered}
$$

Therefore, the total error in measuring $\left\langle M^{2}\right\rangle$ is

$$
\varepsilon=O\left(\sum_{i=0}^{l} s_{i}^{-1 / 2} h_{i}^{3}\left(\pi^{2}+m^{2} h_{i}^{2}\right)^{-1} L^{-2} T\right)
$$

\section{APPENDIX C. CALCULATION OF THE DISCRETIZATION ERROR $\left\langle M^{2}\right\rangle-\left\langle M_{h}^{2}\right\rangle$}

To calculate the discretization error, observe first that for $N / 2<j<N$ the term in $(9 b)$ is smaller than

$$
\frac{T h^{4}}{L^{3}\left(2+m^{2} h^{2}\right)} \leqslant \min \left(\frac{T h^{4}}{2 L^{3}}, \frac{T h^{2}}{m^{2} L^{3}}\right)
$$


hence the sum of all these $N / 2$ terms is less than

$$
C L T \min \left(\frac{1}{N^{3}}, \frac{1}{m^{2} L^{2} N}\right)
$$

where $C=0.5$. A similar estimate, but with a different value of $C$, is obtained for the sum of all terms $N / 2<j<\infty$ in $(5 b)$.

For $j<N / 2$, each term in $(9 b)$ can be approximated by a Taylor expansion as follows:

$$
\begin{aligned}
\frac{4 L T}{\pi^{2}} & \frac{1-(j \pi h / 2 L)^{2}}{\pi^{2} j^{4}\left[1-\frac{2}{3}(j \pi h / 2 L)^{2}\right]+m^{2} L^{2} j^{2}\left[1-\frac{1}{3}(j \pi h / 2 L)^{2}\right]} \\
& =\frac{4 L T}{\pi^{2}} \frac{1-\beta(j \pi h / 2 L)^{2}}{\pi^{2} j^{4}+m^{2} L^{2} j^{2}}
\end{aligned}
$$

where $1 / 3<\beta<2 / 3$. Comparing this with the $j^{\text {th }}$ term in $(5 b)$, we conclude that the total discretization error for these terms is approximately

$$
L T \beta N^{-2} \sum_{j=1}^{N / 2-1} \frac{1}{\pi^{2} j^{2}+m^{2} L^{2}} \leqslant C L T \min \left(\frac{1}{N^{2}}, \frac{1}{m^{2} L^{2} N}\right)
$$

For general $p$-order discretization, a similar estimation would give

$$
C L T \min \left(\frac{1}{N^{p}}, \frac{1}{m^{2} L^{2} N}\right)
$$

Therefore, the total discretization error is

$$
C L T \min \left(\frac{1}{N^{\min (3, p)}}, \frac{1}{m^{2} L^{2} N}\right) \leqslant C L T N^{-\min (3, p)}
$$

hence the relative (to $\sigma$ ) discretization error estimator is

$$
\text { r.d.e. }=\frac{C\left(\pi^{2}+m^{2} L^{2}\right)}{N^{p_{*}}+m^{2} L^{2} N}
$$

where $p_{*}=\min (3, p)$.

Clearly, there is no advantage in raising the order of the discretization error beyond $p=3$.

\section{ACKNOWLEDGMENTS}

This research has been supported in part by grants G0289-065.07/93 from the German-Israeli Foundation for Research and Development 
(GIF), 379/93 from the Israeli Academy of Science and Humanities, and F49620-92-J-0439 from the U. S. Air Force, and by the Carl F. Gauss Minerva Center for Scientific Computation.

\section{REFERENCES}

1. A. Brandt, Multilevel computations: Reviews and recent developments, in Preliminary Proceedings 3rd Copper Mountain Conference on Multigrid Methods (April 1987); see also S. F. McCormick, ed., Multigrid Methods: Theory Applications and Supercomputing (Marcel Dekker, New York, 1988), pp. 35-62.

2. A. Brandt, The Weizmann Institute research in multilevel computation: 1988 report, in Proceedings 4th Copper Mountain Conference on Multigrid Methods, J. Mandel et al., eds. (SIAM, 1989), pp. 13-53.

3. A. Brandt, Multigrid methods in lattice field computations, Nucl. Phys. B (Proc. Suppl.) 26:137-180 (1992).

4. A. Brandt, M. Galun, and D. Ron, Optimal multigrid algorithms for calculating thermodynamic limits, J. Stat. Phys. 74:313-348 (1994).

5. A. Brandt, D. Ron, and D. J. Amit, Multi-level approaches to discrete-state and stochastic problems, in Multigrid Methods, W. Hackbusch and U. Trottenberg, eds. (SpringerVerlag, 1986), pp. 66-99.

6. M. Galun, Optimal multigrid algorithms for model problems in statistical mechanics, M.Sc. thesis, Weizmann Institute of Science (1992).

7. J. Goodman and A. D. Sokal, Multigrid Monte Carlo methods for lattice field theories, Phys. Rev. Lett. 56:1015-1018 (1986).

8. D. Kandel, E. Domany, and A. Brandt, Simulations without critical slowing down-Ising and 3-state Potts models, Phys. Rev. B 40:330 (1989).

9. D. Kandel, E. Domany, D. Ron, A. Brandt, and E. Loh, Jr., Simulations without critical slowing down, Phys. Rev. Lett. 60:1591 (1988).

10. G. Mack and A. Pordt, Convergent perturbation expansions for Euclidean quantum field theory, Commun. Math. Phys. 97:267 (1985); G. Mack, In Nonperturbative Quantum Field Theory, G. t'Hooft et al., eds. (Plenum Press, New York, 1988), p. 309

11. D. Ron, Development of fast numerical solvers for problems in optimization and statistical mechanics, Ph.D. thesis, Weizmann Institute of Science (1989).

12. R. H. Swendsen and J. S. Wang, Nonuniversal critical dynamics in Monte Carlo simulations, Phys. Rev. Lett. 58:86-88 (1987).

13. U. Wolff, Collective Monte Carlo updating for spin systems, Phys. Rev. Lett. 62:361-364 (1989). 